\title{
Study on Grade Prediction of Flotation Concentrate Based on Modified Artificial Fish Swarm Algorithm
}

\author{
Yong Zhang ${ }^{1,{ }^{*}}$, Hongyang Ren ${ }^{1}$ and Zhongfeng $\mathrm{Li}^{2}$ \\ ${ }^{1}$ University of Science and Technology Liaoning, Liaoning Anshan, 114051, China \\ ${ }^{2}$ Yingkou Institute of Technology, Liaoning Yingkou, 115014, China \\ ${ }^{*}$ Corresponding author
}

Keywords: Artificial fish swarm algorithm, Flotation, Support vector machine.

\begin{abstract}
Flotation is a process with nonlinear, large lag, strong coupling and other characteristics, concentrate grade is an important economic index to test the flotation production process, and it is difficult to establish accurate mathematical model, accurate prediction of concentrate grade is needed, which is of great significance to the quality prediction of flotation process. Support vector machine regression prediction was used as basic model, and modified basic artificial fish swarm algorithm(AFSA) was used to optimize parameters about the model, and GAFSA-SVM model was established to predict flotation concentrate grade. The initial value, crowding factor and visual field of the AFSA are improved, and the elimination mechanism is added.By simulating the historical data, the improved gafsa-svm model can predict the flotation concentrate grade well, effectively improve the prediction accuracy and speed of concentrate grade, and meet the requirements of field practice.
\end{abstract}

\section{Introduction}

Flotation is the method of ore dressing in most of China's concentrators. Flotation has a wide range of application fields, fine particle size of flotation ore, high production index and a variety of flotation concentrates, and has a good development prospect. However, there are many physical and chemical changes in the process of flotation, with complex mechanism of action and many influencing factors, such as ore particle size, concentration of pulp, dosage of agents, concentration of foam and temperature. Concentrate grade is not only a parameter of the flotation process, but also an important index to test the flotation efficiency. Soft measurement method can obtain some physical quantities that cannot be measured by instruments, and predict some physical quantities that cannot be measured by establishing mathematical models. . In recent years, some progress has been made in the application of soft-sensing method to flotation prediction. Now there are many soft-sensing modeling methods based on intelligent algorithms. TongXi [1] by optimal `ESN chaos time series RBF neural network to flotation prediction analysis of economic indicators. Zhang Xong, Zhu Jing [2], proposed the economic and technical index prediction of flotation process based on the combination of chaotic ant colony and least square method and simultaneously training neural network.

Li Xiaolei proposed a new method - AFSA that about animal autonomy[3], which is an application of the idea of swarm intelligence and a bottom-up optimization method. AFSA includes random behavior, foraging behavior, clustering behavior and rear-end behavior, through which optimization is carried out. At present, the research of artificial fish swarm algorithm has penetrated into various application fields and obtained good practical application .Cui Qiang[4] et al. applied the AFSA to fault diagnosis of transformer. By improving the AFSA to train the neural network, the accuracy of transformer fault diagnosis was effectively improved. Zhu Xuhui[5] et al. optimized the SVM's parameters by improving the AFSA, established the haze prediction model of the support vector, and proved the effectiveness and stability of the model through experimental data. Aiming at the problems of slow convergence speed and limited convergence precision in the algorithm later stage, the algorithm is improved. The model should be used to predict the flotation concentrate grade based on the improved AFSA. The results of simulation show that the prediction of flotation concentrate grade has higher prediction accuracy, practical significance and meets the requirements of field practice. 


\section{Introduce the Support Vector Machine Regression(SVR)}

SVM can be well applied to the regression estimation problem, and retains all the characteristics of the maximum interval algorithm: the nonlinear function can be obtained by the linear learner in the kernel eigenspace, and the capacity of the system is controlled by parameters unrelated to the dimension of the eigenspace. Like the classification algorithm, the learning algorithm minimizes a convex function and has sparse solutions.

There is a data sample $\left(\mathrm{x}_{1}, \mathrm{y}_{1}\right),\left(\mathrm{x}_{2}, \mathrm{y}_{2}\right), \ldots,\left(\mathrm{x}_{\mathrm{n}}, \mathrm{y}_{\mathrm{n}}\right)$, Then its regression function is expressed as:

$$
\mathrm{f}(\mathrm{x})=\mathrm{w} \cdot \mathrm{x}+\mathrm{b}
$$

Considering the allowable error, the above equation becomes, when relaxation factors $\xi_{\mathrm{i}}$ :

$$
\left\{\begin{array}{c}
\min \frac{1}{2}\|w\|^{2}+C \sum_{i=1}^{N} \xi_{i} \\
y_{i}\left(<w, x_{i}>+b\right) \geq 1-\xi, \quad i=(1,2, \ldots, I) \\
\xi_{i} \geq 0 \quad i=(1,2, \ldots, I)
\end{array}\right.
$$

Carry out even transformation on equation (2) to get:

$$
\left\{\begin{array}{c}
\min \frac{1}{2} \sum_{i=1, j=1}^{N} y_{i} y_{j} a_{i} a_{j}<x_{i} x_{j}>-\sum_{j=1}^{N} a_{j} \\
\sum_{i=1}^{N} y_{i} a_{i}=0 \\
0 \leq a_{i} \leq C \quad i=(1,2, \ldots, I)
\end{array}\right.
$$

Then regression function is:

$$
f(x)=\operatorname{sgn} \sum_{i=1}^{N} a_{i} y_{i}<x_{i}, x>+b
$$

As with SVM in pattern recognition, only a small part of $\alpha_{\mathrm{i}}$ and $\alpha_{\mathrm{i}}^{*}$ here is not 0 , and the samples corresponding to them are support vector machines, which are generally samples at the locations where the function changes dramatically[6].For the regression of nonlinear problems, the nonlinear transformation converted into a linear problem that is in high-dimensional space, and then $\mathrm{K}\left(\mathrm{x}_{\mathrm{i}}, \mathrm{x}\right)$ can be used to replace the inner product operation in (regression function), so that the regression function becomes: $f(x)=\operatorname{sgn}\left(\sum_{i=1}^{N} a_{i} y_{i} K\left(x_{i}, x\right)+b\right)$

The parameters about SVM are of great significance to their learning ability and generalization ability, how to optimize the parameters of SVM is an important problem. For radial basis kernel function of SVM, the main parameters have penalty factor $\mathrm{C}$ and nuclear $\mathrm{G}$, penalty factor $\mathrm{C}$ is adjust the proportion of incredible range and empirical risk, can change the penalty factor in the generalization ability of the model and make a trade-off between misclassification rate and parameter G mainly affect the complex degree of sample data in feature space[7].

\section{Introduce the Artificial Fish Swarm Algorithm}

AFSA is a bottom-up optimization mode, which solves problems through the interaction of fish swarm in the environment. Set the state for the current artificial fish and then select a position within its vision. If the food concentration is greater than the food of current state, it will move in its direction. Conversely, reselect the state and then to determine if it is satisfied.

The variables in the AFSA are: artificial fish's total number $\mathrm{N}$, the current state of the individual artificial fish $\mathrm{X}=\left(\mathrm{x} 1, \mathrm{x} 2 \ldots, \mathrm{x}_{\mathrm{n}}\right)($ where $\mathrm{xi}(\mathrm{i}=1,2 \ldots, \mathrm{n}))$, the largest Step of artificial fish swarm movement, artificial fish Visual field, try_number, crowding factor $\delta$, $\operatorname{rand}()$ is a random number.

Then its basic behavior describes [8]: 
(1)Foraging behavior: $X_{j}=X_{i}+$ Visual *Rand() $\quad X_{i}^{t+1}=X_{i}^{t}+\frac{X_{j}-X_{i}^{t}}{\left\|X_{j}-X_{i}^{t}\right\|} *$ Step * Rand ()

(2)Group behavior: $X_{i}^{t+1}=X_{i}^{t}+\frac{X_{c}-X_{i}^{t}}{\left\|X_{c}-X_{i}^{t}\right\|} *$ Step * Rand 0

(3)Rear-end collision: $X_{i}^{t+1}=X_{i}{ }^{j}+\frac{X_{j}-X_{i}^{t}}{\left\|X_{j}-X_{i}{ }^{t}\right\|} *$ Step * Rand ()

(4)Random behavior: $X_{\mathrm{i}}^{t+1}=X_{i}^{t}+$ Visual * Rand()

\section{Improve Artificial Fish Stocks}

For the studying of the standard AFSA, it is found that artificial fish swarm algorithm has the disadvantages of low search accuracy and slow speed in later period of convergence. However, reason leading to this new town is that the AFSA and step size are fixed values, which directly determine the search scope, search speed and accuracy of the artificial fish algorithm. By this way[9] the later of convergence stage about the algorithm, the artificial fish will gather around the optimal extreme point, at this time, if the size of step for the algorithm is large, the artificial fish will wander around the optimal solution and cannot accurately approximate the optimal solution. If the step size is small, it will affect the convergence speed of the AFSA, and then the artificial fish will search near the local extreme point instead of finding the global extreme point. Therefore, the step size and visual field are improved of the basic AFSA, the performance of the algorithm can be effectively improved [10].

Improve

In the clustering behavior, rear-end behavior and foraging behavior of the AFSA, two different artificial fish horizons were set in the three behaviors. The visual field of rear-end behavior and clustering behavior and is assigned to a larger value, which is called visuall. The distance between the artificial fish and the nearest artificial fish is defined as visual field of foraging behavior, which is called visual2. This setting can not only ensure the convergence speed, but also ensure algorithm's accuracy. In this step size, set a variation coefficient $(01)$, then *visual 2 will be used as the step size of AFSA, so that the step size and field of vision of artificial fish swarm can be improved. Also added obsolescence for the AFSA, for improving the convergence speed, after the iteration algorithm to $1 / 2$ number, carries on the comparison to the objective function value, if higher than a value, then eliminate the artificial fish and release the possession of the space, the number of the artificial fish into $\mathrm{N}-1$, thus reducing the computational complexity of artificial fish.

AFSA does not require a high initial value, but if the artificial fish swarm has an appropriate initial value, then the convergence speed of the algorithm will be accelerated. Therefore, we can adopt uniform fish swarm initialization method. Uniform initial value method can cover the global optimal extreme point and improve the speed of convergence.

Crowding factor $\delta$ is to avoid artificial fish falling into local extreme points due to overcrowding, but it also makes artificial fish unable to approach the extreme points in the field of optimal extreme points. Too much crowding factor will affect the clustering behavior of artificial fish, as well as the convergence speed and accuracy. If the crowding factor is too small, the global performance of the algorithm will be affected[11].In the literature, by the author to crowded degree of factor analysis and verification, we can understand its mechanism of action, so, in this article can ignore the crowded degree factor, so that we can reduce complexity of algorithm can also ensure accuracy of convergence speed, and ability of global optimization[12].

In the following experimental data, the time performance of the standard AFSA and the improved AFSA algorithm is compared. In the simulation experiment, the function with the maximum solution is selected as the standard function. In the experiment, the basic parameters are set uniformly, at the same time, different algorithms are selected to test the same standard function. 
Table 1. Function optimization table

\begin{tabular}{c|c|c|c|c}
\hline Function & To explore the area & Theoretical optimal solution & \multicolumn{2}{|c}{ Optimization of time } \\
AFSA & GAFSA \\
\hline$f(x)=\frac{1}{2} e^{-\frac{x^{2}}{2}}$ & $\mathrm{x} \in[-10,10]$ & 0.5 & 1.19 & 0.546 \\
\hline$f(x)=-\left(x^{2}-1\right)^{3}-1$ & $\mathrm{x} \in[-10,10]$ & 0 & 1.06 & 0.506 \\
\hline$f(x, y)=\frac{x y}{2 x^{2} y^{2}+4 x+4 y}$ & $\begin{array}{l}\mathrm{x} \in[0,10] \\
\mathrm{y} \in[0,10]\end{array}$ & 0.105 & 1.118 & 0.632 \\
\hline
\end{tabular}

Table 2. Standard test functions

\begin{tabular}{c|c|c}
\hline The function name & The formula & Solution space \\
\hline Sphere & $F_{1}(x)=\sum_{i=1}^{D} x_{i}^{2}$ & {$[100,-100]^{D}$} \\
\hline Rastrigrin & $F_{2}(x)=\sum_{i=1}^{D}\left(x_{i}^{2}-10 \cos \left(2 \pi x_{i}\right)+10\right)$ & {$[-5,5]^{D}$} \\
\hline
\end{tabular}

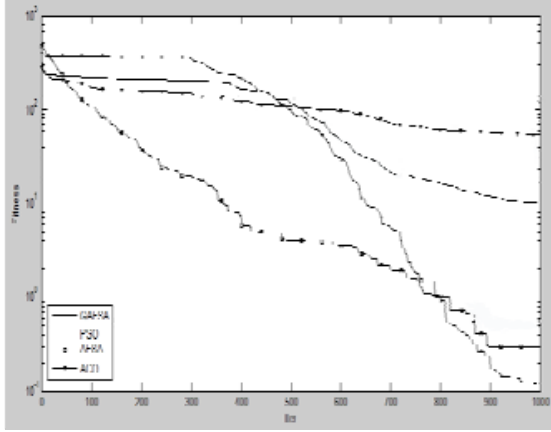

Figure 1. Number of iterations of the Sphere function

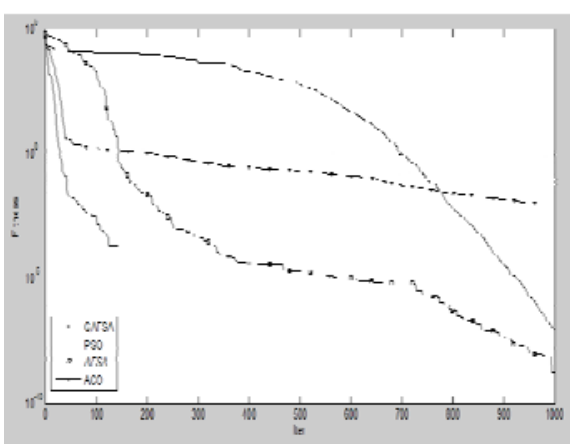

Figure 2. Number of iterations of the Rastrigrin function

As can be seen from the figure, compared with the other three algorithms, the improved AFSA has better convergence accuracy and speed.

\section{Analysis of Experimental Results}

\section{Data Normalization Processing}

In the process of flotation, for on-site data and data, most of them seek for laws through statistical principles and establish learning models to predict future data or some data that cannot be observed. Some data with time series are converted into fitting models with time as independent variable and results as dependent variable for learning and prediction. Because some data do not have direct linear relationship and time variation is relatively small, sometimes it is not ideal to conduct data fitting and merging directly.

Starting from the idea of statistics, the dependent variables and independent variables of data can be normalized to the interval that is conducive to the research, so as to improve the generalization ability. For a sample of data $\{x\}, i=1,2, \cdots, n, x_{n} \in R$, It's normalized to the interval [0,1], Normalized mapping is as follows: $f: x \rightarrow y=\frac{x-\min (x)}{\max (x)-\min (x)} x, y \in R^{n}, x=\left(x_{1}, x_{2}, \cdots, x_{n}\right)$ is the original data in the formula. $\mathrm{y}=\left(y_{1}, y_{2}, \cdots, y_{n}\right)$ is the normalized result of the corresponding $\mathrm{x}, \mathrm{y}_{\mathrm{i}} \in[0,1], i=1,2, \cdots, n, \min (\mathrm{x})$ is minimum of $x, \max (x)$ is maximum of $x ; R$ and $R n$ are represented as 1 and $n$ dimensional sets of real Numbers[13].

\section{SVM Model Parameter Setting}

Establish a regression model for SVM and select radial basis kernel function: $K\left(x_{i}, x_{j}\right)=\exp \left\{-\gamma\left|x_{i} \cdot x_{j}\right|^{2}\right\}$, as the kernel function of the regression model, SVM is of the type of 
SVR, and its loss function value is set as 0.01 , and the penalty factor $C$ is set as $1-100$. The range of nuclear parameter $\mathrm{G}$ is $0.0001 \sim 1$. The number of artificial fish swarm algorithm: $\mathrm{N}=50$, the maximum number of iterations: number $=200$, visual field Visual1 $=10$, change coefficient $\alpha=0.2$, moving Step $=$ $\alpha *$ Visuall.

\section{SVM Model Regression Result Prediction and Error Analysis}

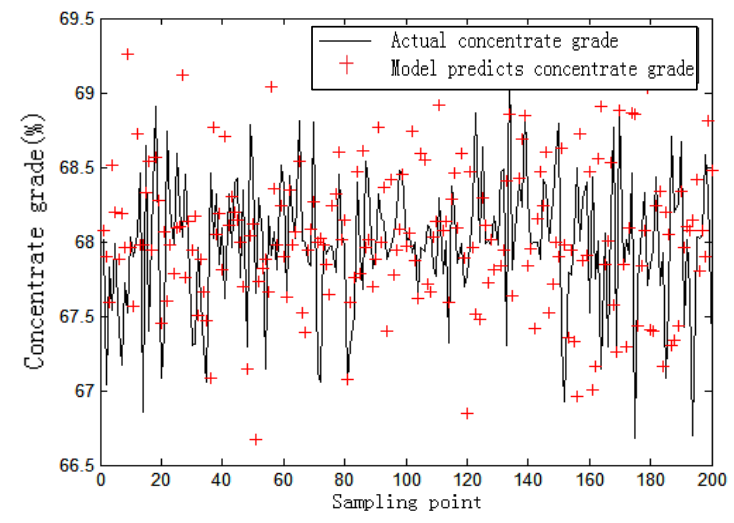

Figure 3. Concentrate grade prediction

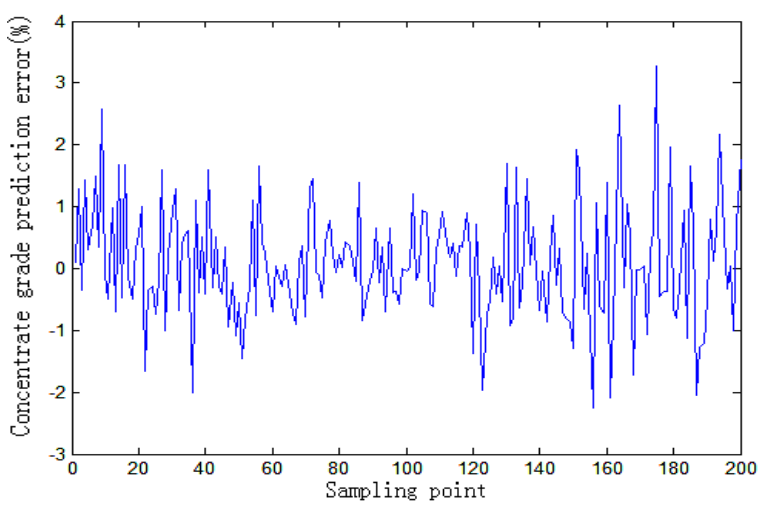

Figure 4. error comparison diagram

\section{Conclusion}

In this article, using SVR as a model, and the AFSA was improved, the improved AFSA has better convergence speed, convergence precision and the overall situation, through the improved artificial fish algorithm of support vector machine (SVM) punishment factor and kernel parameter optimization, and applies the model in mining flotation concentrate grade prediction, the concentrate grade prediction through the historical data, we can see from the simulation results, the optimization model has better prediction precision, able to accurately forecast concentrate grade, prove the validity of the model and practical guiding significance.

\section{Reference}

[1] Tong Xi. Based on the RBF neural ESN Chaotic Time Series Network Analysis for the Forecast of Flotation Economic Indications[D].University of Science and Technology Liaoning.2016

[2] ZHANG Yong ZHU Jing. Forecasting of Economic and Technical Indicators of Flotation Process Based on Chaotic Ant Flotation Neural Network[J].Journal of Shanghai Jiaotong University.2016(6):975-979.

[3] Li Xiaolei. A New Intelligent Optimization Method - Artificial Fish School Algorithm[D].ZGE JIANG UNIVERSITY.2003.

[4] Cui Qiang Li Yinglong Li Zhihong. Transformer Fault Diagnosis Based on Improved Fish Swarm Algorithm and Support Vector Machine[J].Electrical Automation.2017(6):63-66.

[5] Zhu Xuhui Ni Zhiwei. Haze prediction method based on multi-fractal dimension and co-evolution discrete artificial fish swarm algorithm[j].Systems Engineering - Theory \& Practice.2017, 37(4):999-1010.

[6] TIAN Hai-lei LI Hong-ru XU Bao-hua. Support Vector Machine Prediction Based on Improved Artificial Fish Swarm Algorithm[J]. 2013, 39(4):222-225.

[7] LI Su YUAN Zhigao WANG Cong CHEN Tianen GUO Zhaochun. Optimization of support vector machine parameters based on group intelligence algorithm.[J].CAAI Transactions on Intelligent Systems 2018, 13(1):70-84. 
[8] LI Jun Liang Xi-ming. Artificial Fish Swarm Algorithm Convergence Speed Improvement Optimization Simulation.[J].Computer Simulation2018(1):232-238.

[9] LIU Dong-lin LI Le-le. New Improved Artificial Fish Swarm Algorithm[J].Computer Science.2017, 44(4):281-287.

[10]WU Changyou. An improved artificial fish swarm optimization algorithm.[J].CAAI Transactions on Intelligent Systems.2015(3):465-469.

[11]Feng Xiaolin Ning Qian Lei Yinjie Chen Siyu.Support Vector Machine Parameter Optimization Based on Improved Artificial Fish Swarm Algorithm[J]. Computer Measurement \& Control2016, 24(5):237-241.

[12]Kumar K P, Saravanan B, Swarup K S. Optimization of Renewable Energy Sources in a Microgrid Using Artificial Fish Swarm Algorithm[J]. Energy Procedia, 2016, 90:107-113.

[13] Tang Rongzhi.Research on the Method of Data Normalization for Improving SVM Training Efficiency[D].Shandong Normal University. 2016, 31(4):60-65. 